\title{
Der Arbeitskreis Kopf-Hals der Österreichischen Gesellschaft für Ultraschall in der Medizin
}

Der Arbeitskreis Kopf Hals deckt in Österreich die Interessen unterschiedlichster Fachrichtungen ab: Radiologie, Nuklearmedizin, Endokrinologie, Innere Medizin, Chirurgie, HNO, Mund-Kiefer-Gesichtschirurgie und Neurologie.

Diese haben auch unterschiedliche $\mathrm{Zu}$ gänge zu dieser Region. Während einige ihren Schwerpunkt auf der Schilddrüse und den Halslymphknoten haben, liegt dieser bei anderen Fachrichtungen auch auf den übrigen Halsweichteilen, wie Speicheldrüsen, Muskeln, Gefäße oder der Mundhöhle. Ein weiterer, neuer und sich gerade entwickelnder Bereich betrifft die Nerven des Halses. Selbstverständlich überschneidet sich in manchen Bereichen das Arbeitsgebiet des Arbeitskreises mit dem anderer innerhalb der ÖGUM.

Im Arbeitskreis Kopf-Hals trägt wie in Deutschland und der Schweiz ein 2-Stufenkonzept diesen Anforderungen Rechnung und ist die Basis für das „Zertifikat Sonografie der Österreichischen Ärztekammer“, das von den neuen Arbeitskreisleitern Georg Zettinig und Stefan Meng überarbeitet wurde.

Der mindestens 8 Stunden umfassende Grundkurs Kopf-Hals ist die sonografische Grundausbildung für alle Sonderfächer der topografischen Region „Kopf/Hals“. Zumindest 8-stündige Aufbaukurse haben ihren Fokus im jeweiligen Spezialgebiet.

Praktische Übungen im erforderlichen Ausmaß sowie ein Abschluß-Testat sind integrativer Teil jedes Kurses.
Grundkurs Kopf / Hals-Schilddrüse, $8 \mathrm{~h}$ :

- Technik

- Anatomie Kopf-Hals

- Normalbefunde im Hals

- Schilddrüse - diffuse Veränderungen und Knoten

- praktische Übungen

Aufbaukurs Schilddrüse, 8 h:

- Update Technik und Anatomie

- Dignitätsbeurteilung

- Ultraschallgezielte Feinnadelpunktion

- Schilddrüsenkarzinom

- Halslymphknoten

- Nebenschilddrüse

Aufbaukurs Kopf-Hals, 8 h:

- Update Technik und Anatomie

- Halslymphknoten

- Raumforderungen und Entzündungen der Halsweichteile

- Speicheldrüse

Die Absolvierung von Grund- und Aufbaukurs sowie der dokumentierte Nachweis der entsprechenden eigenständig, supervidiert durchgeführten Ultraschalluntersuchungen (Kopf/Hals exkl. Schilddrüse: 200 bzw. Schilddrüse: 150) berechtigt zum Erwerb des jeweiligen Zertifikats Sonografie (Kopf/Hals bzw. Schilddrüse) der Österreichischen Ärztekammer und entspricht auch den ÖGUM-Richtlinien, in Abgleich mit den Schwestergesellschaften.

AK-Leiter: Univ. Doz. Dr. Georg Zettinig/Dr. Stefan Meng

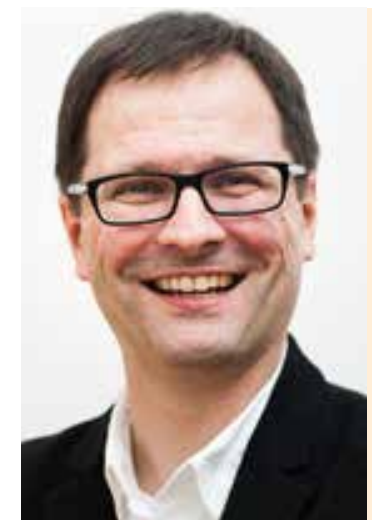

Univ. Doz. Dr. Georg

Zettinig

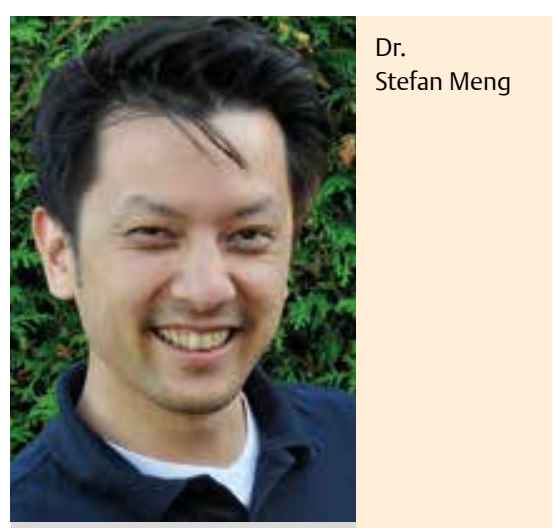

